\title{
An Investigation into the Butyrylcholinesterase-catalyzed Hydrolysis of Formylthiocholine using Heavy Atom Kinetic Isotope Effects
}

\author{
Emily J. Fogle ${ }^{a}$, John F. Marlier ${ }^{a}$, Anthony Stillman ${ }^{b}$, Xin Gao ${ }^{b}$, Yashas Rao ${ }^{b}$, and Lori I. Robins ${ }^{\text {, }}{ }^{*}$ \\ ${ }^{a}$ California Polytechnic State University, San Luis Obispo, CA 93407 and \\ ${ }^{\mathrm{b}}$ University of Washington Bothell, Bothell, WA 98011 \\ *Corresponding author. Irobins@uw.edu
}

\begin{abstract}
Heavy atom kinetic isotope effects (KIEs) were determined for the butyrylcholinesterase-catalyzed hydrolysis of formylthiocholine (FTC). The leaving-S, carbonylC, and carbonyl-O KIEs are ${ }^{34} k=0.994 \pm 0.004,{ }^{13} k=1.0148 \pm 0.0007$, and ${ }^{18} k=0.999 \pm 0.002$, respectively. The observed KIEs support a mechanism for both acylation and deacylation where the steps up to and including the formation of the tetrahedral intermediate are at least partially rate determining. These results, in contrast to previous studies with acetylthiocholine, suggest that the decomposition of a tetrahedral intermediate is not rate-determining for FTC hydrolysis. Structural differences between the two substrates are likely responsible for the observed mechanism change with FTC.
\end{abstract}

Key Words. Butyrylcholinesterase; Kinetic Isotope Effects; Thioester Hydrolysis

\section{Introduction}

Thioesters are energy-rich functional groups that often serve as enzyme substrates for a variety of reactions in metabolism. Many families of proteins utilize thioesters as substrates. For example, acetyl transferases, thiolases, and thioesterases catalyze thioester exchange, Claisen condensations, and hydrolysis of thioesters, respectively. ${ }^{1,2}$ Cholinesterases are one family of enzymes that can be used to study the mechanism of thioester hydrolysis. Although thioesters are not the natural substrate, these enzymes provide insight into the mechanism of thioester hydrolysis and can provide valuable information for the design of transition state inhibitors.

Cholinesterases, such as butyrylcholinesterase (BChE) and acetylcholinesterase ( $\mathrm{AChE})$, are serine hydrolases with the $\alpha / \beta$ hydrolase fold. While the exact role played by BChE is still unknown, it is thought to be primarily responsible for detoxification reactions in the serum, liver, lungs, and intestinal mucosa. ${ }^{3}$ It is also found in the brain and is believed to support acetylcholinesterase in hydrolyzing acetylcholine at the synapse. ${ }^{4}$ Enzymes in this family have active sites that consist of a catalytic triad. They follow a mechanism similar to that of a serine protease in which the activated serine attacks the acyl-substrate to form an acyl-enzyme intermediate. The acyl-enzyme intermediate then reacts directly with a water molecule at the active site. Structural and kinetic data provide strong evidence that both the acylation and deacylation reactions occur through a tetrahedral intermediate. ${ }^{5,6}$ In the current paper we report the results of a continuing heavy atom kinetic isotope effect investigation of BChEcatalyzed thioester hydrolysis. The substrate for these investigations was formylthiocholine and the measured KIEs include the leaving-S, the carbonyl-C, and the carbonyl-O. 


\section{Theory and Calculations}

KIEs are reported as a ratio of rate constants for molecules containing different isotopes at a particular position. For example, a carbon KIE is the ratio of ${ }^{12} k /{ }^{13} k$ and is written as ${ }^{13} k_{\text {obs. }}{ }^{7}$ Rather than a single rate constant, the observed KIE is the empirical KIE and can be influenced by a variety of partially rate-determining steps. In this study the KIEs were measured using natural abundance carbon, oxygen, and sulfur. After a partial reaction, the final isotopic analysis was completed by an isotope ratio mass spectrometer (IRMS) and was reported as a per mil difference $(\delta)$ of the sample relative to that for a standard material.

All KIEs in this paper are measured using the competitive method. ${ }^{8}$ To calculate the KIE from the $\delta$ values three data points are necessary: (a) the $\delta$ for the unreacted substrate or completely reacted product $\left(\delta_{0}\right)$; (b) the $\delta$ for the isolated starting material or product from the partial reaction $\left(\delta_{\mathrm{s}}\right.$ or $\delta_{\mathrm{p}}$ ); and $(\mathrm{c})$ the fraction of reaction. The KIE can be calculated using substrate or product analysis with eq 1 and eq 2, respectively.

$\left.K I E=\log (1-f) /\left[(1-f)\left(\delta_{\mathrm{s}} / 1000+1\right)\right) /\left(\delta_{\mathrm{o}} / 1000+1\right)\right] \quad$ eq 1

$K I E=\log (1-f) /\left[1-\left(f\left(\delta_{\mathrm{p}} / 1000+1\right) /\left(\delta_{\mathrm{o}} / 1000+1\right)\right)\right] \quad$ eq 2

\section{Results and Discussion.}

The family of cholinesterases includes acetylcholinesterase and butyrylcholinesterase. This family of enzymes has an acylation reaction where the acyl substrate is covalently bound to the enzyme and a deacylation reaction where water acts as a nucleophile to hydrolyze the acylenzyme intermediate (see Figure 1). BChE was chosen for this study because substrate inhibition with FTC does not occur as observed in AChE, simplifying analysis.

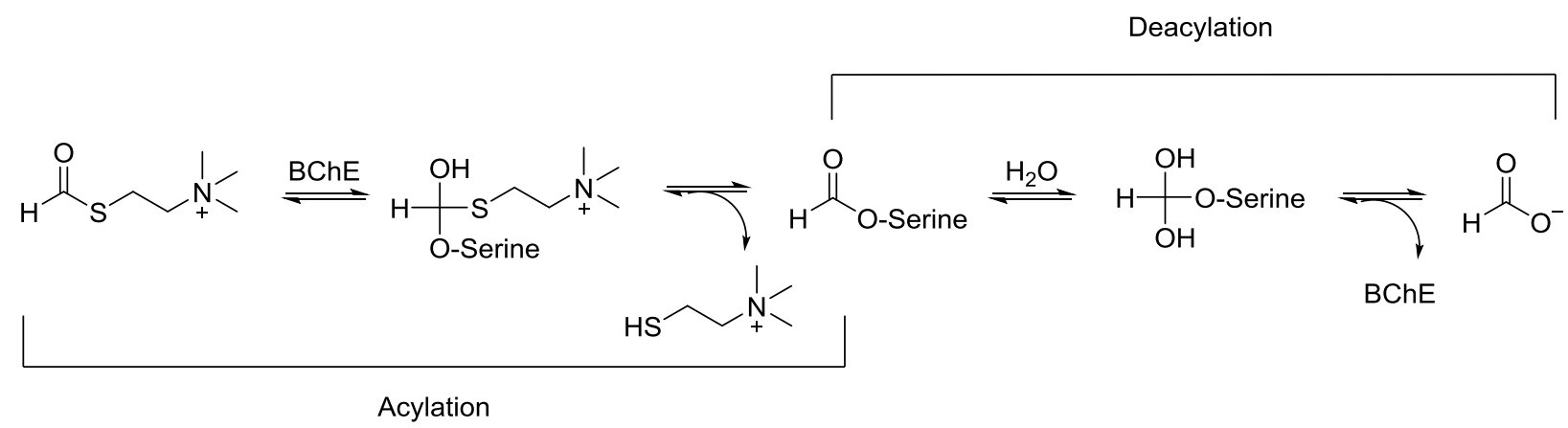

Figure 1. Mechanism of BChE-catalyzed hydrolysis of FTC.

The mechanism of BChE-catalyzed hydrolysis of acetylthiocholine (ATC) was studied previously using KIE experiments. Quinn et al. used the direct method to independently measure the rates of the natural abundance and isotopically enriched substrates. ${ }^{9}$ This method allows determination of both the KIE on $\mathrm{k}_{\text {cat }} / \mathrm{K}_{\mathrm{m}}$ and $k_{\text {cat }}$. Using acetyl- ${ }^{2} \mathrm{H}_{3}$-thiocholine, a secondary $\mathrm{KIE}$ of ${ }^{D 3}\left(k_{\text {cat }} / K_{m}\right)=0.98$ was found. KIEs on $k_{\text {cat }} / K_{m}$ include only steps up to the first irreversible step 
(i.e. product release for acylation). ${ }^{10}$ This small inverse secondary KIE led to the conclusion that some step (or steps) other than chemical formation of the tetrahedral intermediate were partially rate determining. The observed KIE on $k_{\text {cat }}$ was ${ }^{D} k_{\text {cat }}=1.10 .{ }^{10} \mathrm{KIEs}$ on $\mathrm{k}_{\text {cat }}$ measure the effects on the steps after formation of the ES complex through product release. Quinn attributed this normal KIE as arising from an $\mathrm{sp}^{3}$-hybridized carbon in a tetrahedral intermediate going through an $\mathrm{sp}^{2}$-like transition state. This lead to the unusual conclusion that breakdown of a tetrahedral intermediate to an acyl product is rate determining and further implies that a tetrahedral intermediate accumulates during the reaction under saturating conditions. Although unusual, the conclusion is consistent with x-ray crystal structures that find a stable tetrahedral intermediate with the human enzyme, stabilized by a three-pronged oxyanion hole in the BChE active site. ${ }^{6}$ Quinn's, et al., work with equine serum BChE could not determine which of the two tetrahedral intermediates accumulates, although subsequent work with Drosophila melanogaster AChE was consistent with accumulation of the tetrahedral intermediate in the second, deacylation reaction. ${ }^{5}$

Several KIEs for BChE-catalyzed hydrolysis of FTC have been measured previously in our labs using the competitive method, which measures only $\mathrm{k}_{\text {cat }} / \mathrm{K}_{\mathrm{m}}$ isotope effects (Table 1$) .{ }^{11}$ In these studies FTC was used as the substrate instead of ATC. The reason for choosing FTC was the existence of analytical procedures for formate which allowed measurement of multiple KIEs. These $\mathrm{K}_{\text {cat }} / \mathrm{K}_{\mathrm{m}} \mathrm{KIEs}$ fall into two categories. The carbonyl-C, carbonyl-O, formyl-H and leaving-S KIEs provide mechanistic information up to release of thiocholine (the acylation reaction), whereas the nucleophile-O KIE provides mechanistic evidence only for the deacylation reaction.

The magnitude of observed formyl-H KIEs on FTC hydrolyses range from 0.89 to 0.75 (Table 1). In addition, calculated values for a model reaction indicate that very early transition states (little hybridization change at the carbonyl-C) should yield a formyl-H KIE near 0.98, whereas very late transition states will yield values near $0.75 .{ }^{12}$ As a result the observed small inverse formyl-H KIE of ${ }^{D}\left(\mathrm{k}_{\mathrm{cat}} / \mathrm{K}_{\mathrm{m}}\right)=0.89$ for BChE-catalyzed hydrolysis of FTC is consistent with a somewhat early transition state that occurs during rate determining formation of the tetrahedral intermediate. A second possibility is that formation of the tetrahedral intermediate is only partially rate determining and is masked by other prior steps (e.g. binding).

The observed nucleophile-O KIE was small and inverse $\left({ }^{18} k=0.9925\right) .{ }^{11}$ If it is assumed that the oxygen equilibrium isotope effect for partitioning water between bulk solvent and the active site of the enzyme is negligible, the observed nucleophile-O KIE will then only report on the deacylation step. It is during deacylation that water is a substrate for the hydrolysis of the acyl enzyme, resulting in all the significant bonding changes to the nucleophile-O. If breakdown of the tetrahedral intermediate in the deacylation step is rate determining, a large inverse KIE (due mostly to the ${ }^{18} K_{\text {eq }}$ for formation of the tetrahedral intermediate) would be expected. ${ }^{13}$ Since the observed KIE is only slightly inverse, steps up to and including formation of the tetrahedral intermediate for the deacylation step are at least partially rate determining.

Table 1. Kinetic isotope effects for the butrylylcholinesterase catalyzed and non-enzymatic hydrolysis of FTC. 


\begin{tabular}{|l|l|l|l|l|l|}
\hline & Leaving-S & Carbonyl-C & Carbonyl-O & Formyl-H & Nucleophile-O \\
\hline BChE & $0.994 \pm 0.004$ & $1.0148 \pm 0.0007$ & $0.999 \pm 0.002$ & $0.89 \pm 0.02^{\mathrm{a}}$ & $0.9925 \pm 0.0008^{\mathrm{a}}$ \\
\hline $\begin{array}{l}0.20 \mathrm{M} \mathrm{HCl} \\
(\mu=0.20)\end{array}$ & $0.995 \pm 0.002^{\mathrm{b}}$ & $1.0312 \pm 0.0009^{\mathrm{b}}$ & $0.997 \pm 0.002^{\mathrm{b}}$ & $0.80 \pm 0.02^{\mathrm{a}}$ & N/A \\
\hline $\begin{array}{l}\text { neutral H2O } \\
(\mu=0.20)\end{array}$ & $0.996 \pm 0.003^{\mathrm{b}}$ & $1.022 \pm 0.001^{\mathrm{b}}$ & $1.010 \pm 0.004^{\mathrm{b}}$ & $0.75 \pm 0.02^{\mathrm{a}}$ & $0.9917 \pm 0.0009^{\mathrm{a}}$ \\
\hline $\begin{array}{l}0.0050 \mathrm{M} \\
\mathrm{NaOH} \\
(\mu=0.20)\end{array}$ & $1.000 \pm 0.001^{\mathrm{b}}$ & $1.0263 \pm 0.0008^{\mathrm{b}}$ & $0.992 \pm 0.003^{\mathrm{b}}$ & $0.88 \pm 0.02^{\mathrm{a}}$ & $\begin{array}{l}1.029 \pm 0.001 \\
\left(\mathrm{H}_{2} \mathrm{O}\right) 0.989 \pm \\
0.001\left(\mathrm{HO}^{-}\right)^{\mathrm{a}}\end{array}$ \\
\hline
\end{tabular}

${ }^{\mathrm{a}}$ Data from reference ${ }^{11}$. ${ }^{\mathrm{b}}$ Data from reference ${ }^{14}$.

The leaving-S KIE measured in this study was determined with natural abundance FTC using the competitive method and gives the isotope effect on $\mathrm{k}_{\mathrm{cat}} / \mathrm{K}_{\mathrm{m}}$. The observed inverse leaving-S KIE of 0.994 for BChE-catalyzed hydrolysis is statistically different than unity (>98\% C.I.), but not statistically different than the leaving-S KIEs for the acid-catalyzed and neutral hydrolyses of FTC (also >98\% C.I.) determined previously (Table 1). ${ }^{14,15}$ These inverse S-KIEs are somewhat surprising since leaving group heteroatom KIEs for oxoesters and amides are most often normal. ${ }^{8}$ The inverse sulfur KIEs for the hydrolysis of FTC in neutral and acidic conditions were attributed to new bending modes for the sulfur during formation of the tetrahedral intermediate which leads to stiffer bonding to the sulfur atom and an inverse KIE. This trend toward a more inverse sulfur KIE in later transition states was also observed with phosphothioate ester hydrolysis. ${ }^{15}$ In the present case of $\mathrm{BChE}$-catalyzed hydrolysis, the inverse leaving-S KIE leads to the conclusion that breakdown of the tetrahedral intermediate during acylation is not rate determining, since rate-determining breakdown of the tetrahedral intermediate should give rise to a normal KIE. Because accumulation of the tetrahedral intermediate requires its breakdown to be rate determining, this also means that the tetrahedral intermediate for acylation does not accumulate in the hydrolysis of FTC. However, the reason for the observed inverse effect must be different than the non-enzymatic cases where late $\mathrm{sp}^{3}$-like transition states are proposed on the basis of larger inverse formyl-H observed KIEs. ${ }^{14}$ For BChE catalysis the inverse leaving-S KIE may be due to partially ratedetermining steps prior to formation of the tetrahedral intermediate that lead to stiffer bonding to the sulfur atom. Such steps may include formation of partial bonds between the sulfur and active site residues prior to formation of the bond between the carbonyl-C of FTC and the serine oxygen. The results of the leaving-S KIE together with the formyl-H and nucleophile-O KIEs indicate that steps leading up to and including formation of the tetrahedral intermediate are at least partially rate determining in both the acylation and deacylation steps in the BChE-catalyzed hydrolysis of FTC. ${ }^{11}$

The KIEs determined with FTC as a substrate do not support the accumulation of a tetrahedral intermediate as observed with ATC. Formyl thioesters are considerably more reactive toward nucleophiles than acetyl thioesters and this should lower the transition state energies for both formation and decomposition of the tetrahedral intermediate in the acylation step. In turn this might cause steps prior to attack by the serine hydroxyl (such as binding) to become (more) partially rate determining. To explain the small inverse observed formyl-H and leaving-S KIEs 
these prior steps must not significantly affect the hybridization of the carbonyl-C while simultaneously stiffening the bonding at sulfur.

The observed carbonyl-C KIE for the BChE-catalyzed reaction is ${ }^{13}\left(\mathrm{k}_{\mathrm{cat}} / \mathrm{K}_{\mathrm{m}}\right)=1.0148$. In theoretical studies on addition of hydroxide to acetaldehyde (a mimic for formation of a tetrahedral intermediate) it was shown that the carbonyl-C KIE decreases in the direction of later transition states. A maximal KIE of 1.031 is expected for a very early transition state and 1.006 for a very late one. ${ }^{12}$ For the non-enzymatic hydrolysis of FTC the observed carbonyl-C KIEs are all greater than 1.022; the hydrolysis of oxoesters gives KIEs of similar magnitude. ${ }^{8}$ The observed smaller magnitude of the carbonyl-C KIE on the BChE-catalyzed hydrolysis would seem to suggest a later transition state than those observed in acidic and neutral hydrolyses. This is inconsistent with the observed smaller inverse formyl-H KIE for BChE catalysis (0.89) versus those for acidic (0.80) and neutral hydrolysis (0.75). However, if steps prior to formation of the bond between the nucleophile and the carbonyl-C are partially rate determining in the enzymatic case, both a smaller normal observed carbonyl-C KIE and a smaller inverse formyl-H KIE would be expected. This is consistent with the interpretation above.

The carbonyl-O KIE is more difficult to interpret in this reaction. The observed carbonyl-O KIE for the $B C h E$-catalyzed reaction is ${ }^{18}\left(\mathrm{k}_{\text {cat }} / \mathrm{K}_{\mathrm{m}}\right)=0.999$ and is not statistically different from unity. Aforementioned theoretical calculations indicate that the magnitude of the carbonyl-O KIE increases from a minimum of 1.004 for early transition states to a maximum of 1.032 in late transition states. ${ }^{12}$ The carbonyl-O is influenced by 1 ) the loss of the $\pi$-bond to the carbonyl-O which gives a normal KIE, 2) formation of new bonds to hydrogen atoms which can give a normal or inverse contributions to the KIE and 3) new bending and torsional modes in the transition state which provides inverse contributions to the KIE. In the case of BChE, normal and inverse contributions are balanced. It is most likely that the inverse contributions are due

to increasing interactions between the carbonyl-O and stabilizing residues in the oxyanion hole in the transition state while the normal contributions are due to the loss of the $\pi$-bond to the carbonyl-O. Furthermore, the already small magnitude of this KIE may be reduced by prior partially rate-determining steps leading to the observed value of unity.

\section{Conclusion}

The BChE-catalyzed hydrolysis of FTC follows a stepwise mechanism involving formation of a tetrahedral intermediate. Unlike ATC, there is no evidence for the accumulation of a tetrahedral intermediate during the reaction. The result of the leaving-S KIE experiment suggests stiffer bonding to the sulfur in the transition state for acylation and supports previous studies that steps prior to formation of the tetrahedral intermediate in the acylation step are at least partially rate determining. The carbonyl-O KIE is consistent with interactions between the substrate and the oxyanion hole, which are well established structurally. The unusually small carbonyl-C KIE supports the hypothesis that steps prior to the actual attack of the nucleophile are partially rate determining.

\section{Experimental}


6.1 Synthesis of Formylthiocholine. Formylthiocholine was synthesized following literature procedures. $^{11}$

6.2 Leaving-S KIE Procedures for Butyrylcholinesterase. The procedure used to determine the leaving-S KIE were based on a previously published protocol. ${ }^{14}$ Briefly, formylthiocholine (14 $\mathrm{mg}, 51.1 \mu \mathrm{mol})$ was added to $1 \mathrm{~mL}$ of $50 \mathrm{mM}$ 2-(N-morpholino)ethanesulfonic acid (MES) buffer at $\mathrm{pH}$ 6.8. The reaction was initiated by the addition of equine serum BChE (Sigma Aldrich C1057) to a final concentration of $14 \mathrm{nM}$. The lyophilized enzyme obtained from the manufacturer was used without further purification, as the exact concentration of the enzyme is not required when using the competitive method to determine the $\mathrm{k}_{\mathrm{cat}} / \mathrm{Km} \mathrm{KIE}$. The reaction was monitored using the decrease in absorbance at $285 \mathrm{~nm}$ due to FTC hydrolysis. After the reaction was $\sim 50 \%$ complete, the exact fraction of reaction was determined using the DTNB assay. ${ }^{16}$ The reaction was immediately added to $10 \mathrm{~mL}$ of $50 \mathrm{mM}$ MES buffer at pH 6.8 containing 1.1 equivalent (5,5'-dithiobis-(2-nitrobenzoic acid) (DTNB) $(22 \mathrm{mg}, 55.5 \mu \mathrm{mol}$ ) added. The solution was immediately loaded onto a cation-exchange column ( $2 \mathrm{~mL}$ of BT AG $50 \mathrm{~W}-\mathrm{X} 8$ Resin BIO-RAD hydrogen resin). The column was washed with $20 \mathrm{~mL}$ of water and the starting FTC was eluted with $0.5 \mathrm{M}$ triethylammonium bicarbonate (TEAB) at pH 8.5 into $6 \mathrm{~mL}$ fractions. The fractions were assayed with DTNB to determine those that contained thiol. Those with significant absorbance at $412 \mathrm{~nm}$ were collected and the buffer was removed by rotary evaporation. The samples were further dried by lyophilization and were analyzed by isotope ratio mass spectrometry (IRMS). Controls were performed in which FTC was treated as above without the addition of BChE. After application to the cation exchange column fractions containing FTC were collected, concentrated and submitted for IRMS analysis. This ${ }^{34} \delta$ was used for the leaving-S KIE calculations and was within standard error of the original FTC.

Controls: (a) FTC was completely hydrolyzed to formate and thiocholine with $\mathrm{NaOH}$. The resulting solution was neutralized, treated with $22 \mathrm{mg}$ of DTNB and applied to the cation exchange column. The column was washed with water, followed by elution with $0.5 \mathrm{M} \mathrm{TEAB}$ at $\mathrm{pH}$ 8.5. No sulfur-containing compounds were detectable in the TEAB fractions via combustion analysis by IRMS. (b) FTC was treated with 1.1 equivalent of DTNB. This solution was added to the cation exchange column. The column was washed with water and the FTC was eluted with $0.5 \mathrm{M}$ TEAB at $\mathrm{pH}$ 8.5. After removal of water, the ${ }^{34} \delta$ of the isolated FTC was determined by IRMS $\left({ }^{34} \delta=19.8 \pm 1.6\right)$ and compared to that of FTC that was not applied to the column $\left({ }^{34} \delta=\right.$ $20.3 \pm 1.8)$. These controls assured no fractionation occurs during the isolation and analysis procedures and that there are no extraneous sources of sulfur present during elution of FTC.

6.3 Carbonyl-C and Carbonyl-O Procedures. The carbonyl-C and carbonyl-O KIEs were measured in a single experiment. The reaction mixture contained $16 \mathrm{mg}$ of FTC (57 $\mu \mathrm{mol})$ in $1000 \mu \mathrm{L}$ of $0.050 \mathrm{M}$ MES buffer at $\mathrm{pH}$ 6.8. A $4 \mu \mathrm{L}$ aliquot of a BChE solution (Sigma Aldrich C1057) was added to initiate the reaction. The final concentration of $\mathrm{BChE}$ in the reaction mixture was $\sim 4.5 \mathrm{nM}$. The reaction was monitored by observing the absorbance change at 285 $\mathrm{nm}$. At the appropriate percent of reaction, the reaction mixture was rapidly chilled to $4{ }^{\circ} \mathrm{C}$, added to a strong anion exchange resin (Biorad $A G \AA 1$ in the acetate form), and rapidly rinsed 
with $30 \mathrm{~mL}$ of water. The product, formate, was bound to the column, while unreacted FTC was rapidly removed in the void volume. The formate was isolated by elution with $0.1 \mathrm{M} \mathrm{NaCl}$ and the $\mu \mathrm{mol}$ of formate in the eluent was determined by a formate dehydrogenase (FDH) assay. The $\mu \mathrm{mol}$ of formate eluted was compared to the original number of $\mu \mathrm{mol}$ of FTC to calculate the fraction of reaction. A $1.5 \mathrm{~mL}$ aliquot of $1.5 \mathrm{M}$ MES buffer at $\mathrm{pH} 6.3$ was added to the combined fractions containing formate. This solution was evaporated to a small volume ( $1 \mathrm{~mL})$ and transferred to a round bottom flask that was equipped with two stopcocks. One stopcock was on a sidearm that was capped with a septum. The second stopcock was for attachment to the high vacuum line. The solution was dried under high vacuum at $70{ }^{\circ} \mathrm{C}$ overnight. While under vacuum, $2 \mathrm{~mL}$ of anhydrous dimethyl sulfoxide (DMSO) containing $250 \mathrm{mg}$ of $\mathrm{I}_{2}$ was added through the sidearm to the dried formate and the resulting $\mathrm{CO}_{2}$ was collected into a liquid nitrogen trap as previously described. ${ }^{11}$ Isotopic analysis gave the $\delta$ for both the oxygen and carbon atoms. The above $\delta$ values and those for formate isolated after complete alkaline hydrolysis were used in the KIE calculation.

Controls: (a) Formate of known isotopic composition was eluted from the anion exchange column with $0.1 \mathrm{M} \mathrm{NaCl}$ in the absence of FTC. The known ${ }^{18} \delta$ of formate did not change after elution. (b) FTC was also passed through the same anion exchange column. FTC was detected in the water wash. However, elution with $0.10 \mathrm{M} \mathrm{NaCl}$ did not produce formate.

Acknowledgements. This work was supported by a grant from the National Science Foundation (CHE-1049689).

(1) Kursula, P.; Ojala, J.; Lambeir, A. M.; Wierenga, R. K. Biochemistry 2002, 41, 15543.

(2) Haapalainen, A. M.; Merilainen, G.; Wierenga, R. K. Trends in Biochemical Sciences 2006, 31, 64.

(3) Johnson, G.; Moore, S. W. Neurochemistry international 2012, 61, 783.

(4) Mesulam, M. M.; Guillozet, A.; Shaw, P.; Levey, A.; Duysen, E. G.; Lockridge, O. Neuroscience 2002, 110, 627.

(5) Tormos, J. R.; Wiley, K. L.; Wang, Y.; Fournier, D.; Masson, P.; Nachon, F.; Quinn, D. M. Journal of the American Chemical Society 2010, 132, 17751.

(6) Nicolet, Y.; Lockridge, O.; Masson, P.; Fontecilla-Camps, J. C.; Nachon, F. J Biol Chem 2003, 278, 41141.

(7) Northrop, D. B. In Isotope Effects on Enzyme-Catalyzed Reactions; Cleland, W. W. O'Leary, M.H.

Northrop, D.B.: University Park Press 1977, p 122.

(8) Marlier, J. F. Accounts of Chemical Research 2001, 34, 283.

(9) Tormos, J. R.; Wiley, K. L.; Seravalli, J.; Nachon, F.; Masson, P.; Nicolet, Y.; Quinn, D. M. Journal of the American Chemical Society 2005, 127, 14538.

(10) Wiley, K. L.; Tormos, J. R.; Quinn, D. M. Chemico-biological interactions 2010, $187,124$. 
(11) Robins, L. I.; Meisenheimer, K. M.; Fogle, E. J.; Chaplan, C. A.; Redman, R. L.; Vacca, J. T.; Tellier, M. R.; Collins, B. R.; Duong, D. H.; Schulz, K.; Marlier, J. F. Journal of Organic Chemistry 2013, 78, 12029.

(12) Hogg, J. L.; J., R.; Kovach, I.; Schowen, R. L. Journal of the American Chemical Society 1980, 102, 79.

(13) Marlier, J. F.; Cleland, W. W. Biochemistry 2006, 45, 9940.

(14) Marlier, J. F.; Fogle, E. J.; Redman, R. L.; Stillman, A. D.; Denison, M. A.; Robins, L. I. Journal of Organic Chemistry 2015, 80, 1905.

(15) Catrina, I. E.; Hengge, A. C. Journal of the American Chemical Society 2003, 125, 7546.

(16) Ellman, G. L.; Courtney, K. D.; Andres, V., Jr.; Feather-Stone, R. M. Biochemical pharmacology 1961, 7, 88. 


$$
{ }^{2}{ }_{\mathrm{k}=0.89}^{18} \mathrm{k}=0.9994
$$

